\title{
Antidepressant-like effect of salidroside and curcumin on the immunoreactivity of rats subjected to a chronic mild stress model
}

\author{
Liliya V. Vasileva ${ }^{\mathrm{a}, \mathrm{e}, *}$, Kremena E. Saracheva ${ }^{\mathrm{a}}$, Mariya V. Ivanovska ${ }^{\mathrm{b}, \mathrm{c}}$, Atanaska P. Petrova ${ }^{\mathrm{b}}$, \\ Andrey S. Marchev ${ }^{\text {d,e }}$, Milen I. Georgiev ${ }^{\text {d,e }}$, Mariana A. Murdjeva ${ }^{\mathrm{b}, \mathrm{c}}$, Damiyanka P. Getova ${ }^{\mathrm{f}}$ \\ a Department of Pharmacology and Drug Toxicology, Faculty of Pharmacy, Medical University Plovdiv, 4000, Plovdiv, Bulgaria \\ ${ }^{\mathrm{b}}$ Department of Microbiology and Immunology, Faculty of Pharmacy, Medical University Plovdiv, 4000, Plovdiv, Bulgaria \\ ${ }^{c}$ Division of Immunological Assessment of Post-traumatic Stress Disorder, Technological Center for Emergency Medicine (TCEMED), Plovdiv, Bulgaria \\ d Group of Plant Cell Biotechnology and Metabolomics, The Stephan Angeloff Institute of Microbiology, Bulgarian Academy of Sciences, 139 Ruski Blvd., 4000, Plovdiv, \\ Bulgaria \\ e Center of Plant Systems Biology and Biotechnology, 4000, Plovdiv, Bulgaria \\ ${ }^{\mathrm{f}}$ Laboratory of Experimental Neuropharmacology, Technological Center for Emergency Medicine (TCEMED), 4000, Plovdiv, Bulgaria
}

\section{A R T I C L E I N F O}

\section{Keywords:}

Salidroside

Curcumin

Fluoxetine

Chronic mild stress

Immunoreactivity

LPS-Induced inflammation

\begin{abstract}
A B S T R A C T
Deregulated cytokines' production is found in depressed patients. Salidroside and curcumin both have been described with potential antidepressant-like activities. The present study investigated the effect of pure salidroside, curcumin and their combination on the immunoreactivity of animals, subjected to a chronic mild stress (CMS) model, followed by lipopolysaccharide (LPS)-induced inflammation. Wistar male rats were separated in the following six groups: control, CMS model, fluoxetine $(2.5 \mathrm{mg} / \mathrm{kg}$, oral), salidroside (5 mg/ kg, oral), curcumin $(20 \mathrm{mg} / \mathrm{kg}$, oral) and salidroside + curcumin $(5 \mathrm{mg} / \mathrm{kg}+20 \mathrm{mg} / \mathrm{kg}$, oral). Changes in glucose preference, spatial learning and exploratory behavior were recorded. The IL- 6 levels in the rats' sera and of the TNF- $\alpha$ levels in the rats' sera and the brain tissue homogenate were evaluated. The groups exposed to stress and treated with fluoxetine, salidroside, curcumin or salidroside + curcumin showed increase in the glucose preference and locomotor activity, as well as, decrease in the escape latency and the cytokines' levels compared to the CMS model group. The chronic stress induced behavioral alternations and increased cytokines' levels in rats which were reversed by administration of salidroside and curcumin, suggesting antidepressant-like effects comparable to that of fluoxetine and potential synergistic interaction regarding the anti-inflammatory and anti-stress effects.
\end{abstract}

\section{Introduction}

Stress is associated with increased risk of psychopathologies as a major depression. In certain subgroups of patients with severe depression increased immune activation and inflammatory rections are characteristic (Hughes et al., 2016). Recent advances in the field of psychoneuroimmunology have identified some mechanisms of interaction between altered immune reactivity and depressive disorders (Miller and Raison, 2015). However, the nature of depression is still inadequately explained since only half of the depressed patients achieve remission with the currently available therapy, which mainly target the monoaminergic signaling pathways (Ménard et al., 2016). Cytokines such as tumor necrosis factor alpha (TNF- $\alpha$ ), interleukins $1 \beta$ (IL-1 $\beta$ ) and 6 (IL-6) are registered in excess in depressed patients with certain phenotypes (Bortolato et al., 2015; Maes et al., 2012; Pariante, 2017).

The stress response mechanisms are involved in the reconstitution of the initial homeostasis or establishment of a new level of homeostasis after successful adaptation, i.e. allostasis (McEwen, 2007). While acute stress induces the fight or flight response and could be beneficial or harmful depending of the intensity of the stressor, chronic stress that persist for weeks, months and longer could only have detrimental effects on health (Dhabhar, 2014). Exposure to chronic stress is confirmed

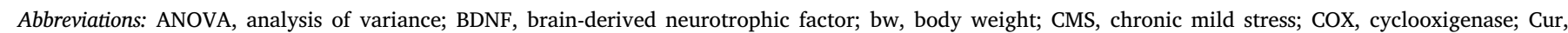

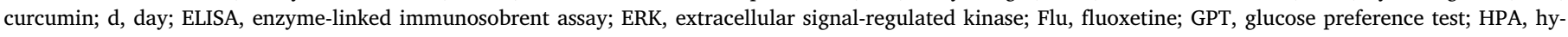

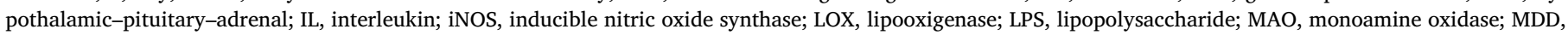

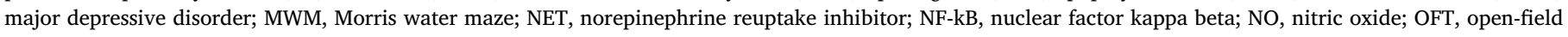
test; Sal, salidroside; SEM, standard error of the mean; SERT, serotonin reuptake inhibitor; TLR-4, Toll-like receptor 4; TNF- $\alpha$, tumor necrosis factor alpha

* Corresponding author. Department of Pharmacology and drug toxicology, Faculty of Pharmacy, Medical University Plovdiv, 4000, Plovdiv, Bulgaria.

E-mail address: vasileva.1.vl@gmail.com (L.V. Vasileva).
} 
to play a causal role in the etiology of the major depressive disorder (MDD) and other depression-like disorders (Maes et al., 2012; Pleil et al., 2012).

The chronic mild stress (CMS) model is among the most accurate and frequently employed models to induce anhedonic-like behavior in rodents, which represents core symptoms of human depression (Willner, 2017, 2005). The bacterial endotoxin, lipopolysaccharide (LPS), when systemically administered, activates the Toll-like receptor 4 (TLR-4) on immune cells and hence provokes the release of a cascade of other cytokines, such as the IL- 6 and the TNF- $\alpha$. The administration of LPS induces depressive-like behavior in rodents accompanied with an activation of the innate immune response (Kubera et al., 2013; Ohgi et al., 2013). Therefore it could be suggested that CMS model followed by a LPS-challenge would have greater effect on the immunoreactivity of depressed rats (Elgarf et al., 2014; Vasileva et al., 2017).

Rhodiola rosea L. (Crassulaceae) is traditionally known as a plant adaptogen which stimulate the nervous system, increase both mental and physical performance and reduce the symptoms of chronic stress and depression (Marchev et al., 2016). Salidroside as among the main bioactive compounds of $R$. rosea has been studied in a number of experimental and clinical studies that confirm its neuroprotective properties (Lee et al., 2013; Shi et al., 2012; Zhang et al., 2013). Zhang et al. (2013) studied the effect of salidroside on an Alzheimer's model and demonstrate a memory-stimulating effect in a water maze task. Possible mechanism suggested by the authors is the modulation of neuroinflammation in the hippocampus by inhibition of the cyclooxygenase 2 (COX-2), the nuclear factor kappa beta (NF-kB) and theinducible nitric oxide synthase (iNOS; Zhang et al., 2013). In a more recent study salidroside is reported to regulate the production of IL- $1 \beta$, IL- 6 and TNF- $\alpha$ both in the serum and in the hippocampus of rats with arthritis-induced cognitive deficits suggesting anti-inflammatory action mediated via the NF-kB pathway (Zhu et al., 2016).

Extracts of Curcuma longa L. (Zingiberaceae) possess wide range of beneficial effects including antidepressant-like and neuroprotective (Goel et al., 2008). The major bioactive compound in the turmeric extract curcumin has well established anti-inflammatory properties as it inhibits the COX enzymes, the lipooxigenase (LOX) enzymes, the production of NO and lowers the levels of some pro-inflammatory cytokines, such as the IL-1 $\beta$ and the TNF- $\alpha$ (Hatcher et al., 2008). A number of recent preclinical studies have shown curcumin to be a potential natural antidepressant in various models of depression (Hurley et al., 2013; Jiang et al., 2013; Tizabi et al., 2014). Although the mechanism of its action need further clarification, it is assumed that curcumin acts as an inhibitor of the enzyme monoamine oxidase (MAO), modulates the release of serotonin and dopamine, regulates the hypothalamic-pituitary-adrenal (HPA) axis activity, elevates the levels of the brain-derived neurotrophic factor (BDNF) and promotes neurogenesis in the hippocampus (Wang et al., 2014).

In our previous set-up chronic administration of the combination of the standardized extracts from $R$. rosea and $C$. longa showed beneficial antidepressant-like effects in rats subjected to the CMS model and influenced the immune response following LPS stimulation (Vasileva et al., 2017). Therefore the present study aimed to further clarify the potential antidepressant-like effects of the pure compounds salidroside, curcumin and their combination on the immunoreactivity of animals subjected to the CMS model followed by a LPS-induced inflammation. Additionally, the results were compared to the effect of the serotonin reuptake inhibitor (SERT) fluoxetine as a positive control.

\section{Materials and methods}

\subsection{Main reagents and kits}

Curcumin ( $\geq 98 \%$ ), fluoxetine hydrochloride ( $\geq 98 \%$ ), salidroside ( $\geq 98 \%$ ) and pentobarbital sodium $(\geq 98 \%)$ were purchased from Sigma-Aldrich (St. Louis, MO, USA). The LPS from Escherichia coli (serotype 055:B5) was supplied from Sigma-Aldrich Chemie GmbH (Steinheim, Germany). The enzyme-linked immunosorbent assays (ELISA) were performed using the following commercial kits: rat IL-6 ELISA kit of Diaclone SAS (Besancon Cedex, France), rat TNF- $\alpha$ ELISA kit of Diaclone SAS (Besancon Cedex, France) and rat TNF- $\alpha$ ELISA kit Abcam (Cambridge, UK).

\subsection{Animals}

Forty eight male Wistar rats weighting from 190 to $230 \mathrm{~g}$ were obtained from the Animal Facility of The Medical University Plovdiv (Plovdiv, Bulgaria). Following randomization into six groups the animals were housed in standard laboratory conditions $\left(23-25^{\circ} \mathrm{C}, 50-55 \%\right.$ humidity, $12 / 12 \mathrm{~h}$ light/dark cycle) and were provided with free access to food and water unless other conditions were stated in the chronic stress protocol.

All procedures in this study were approved by the Bulgarian Food Safety Agency with protocol № 98/22.05.2014, from the Ethic Committee of the Medical University Plovdiv with protocol № 2752/ 09.11.2015 and were carried out following the guidelines of the European Directive 2010/63/EU.

\subsection{Study design}

The chronic mild stress procedure was followed for four weeks to induce depression-like behavior in the experimental animals as described in details previously (Vasileva et al., 2017).

The glucose preference test (GPT) was applied to assess the anhedonic-like behavior induced by the CMS procedure. Changes in the body weight of the animals were recorded weekly.

On the first day after the cessation of the CMS protocol (day 29) the treatment regimen was initiated and lasted for eight days. Behavioral testing of the locomotor activity and spatial memory were started on the 29th day after the administration of the first dose of the respective treatments and lasted until the 36th day. The immunological challenge and the ELISA tests were done at the end of the study on the 37th and 38th day, as illustrated in Fig. 1.

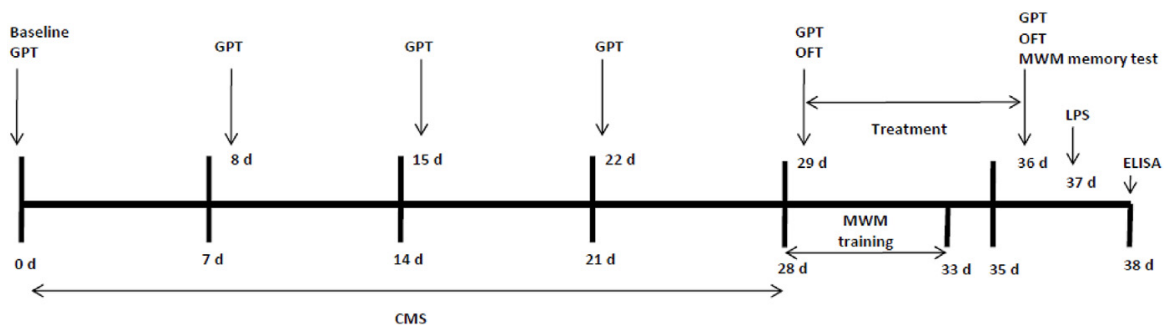

Fig. 1. The outline of the study design (CMS, chronic mild stress; GPT, glucose preference test; ELISA, enzyme-linked immunosorbent assay; LPS, lipopolysaccharide; MWM, Morris water maze; OFT, open field test; d, day). 


\subsection{Treatment regimen}

The animals were treated orally daily for eight days from the 29th day until the 36th day with: distilled water $10 \mathrm{~mL} / \mathrm{kg}$ bw (the control group and the CMS model group); fluoxetine (Flu) $2.5 \mathrm{mg} / \mathrm{kg}$ bw; salidroside (Sal) $5 \mathrm{mg} / \mathrm{kg}$ bw; curcumin (Cur) $20 \mathrm{mg} / \mathrm{kg}$ bw; salidroside $5 \mathrm{mg} / \mathrm{kg}$ bw + curcumin $20 \mathrm{mg} / \mathrm{kg}$ bw. The intragastric administration was done with a stainless steel ball-tipped gavage needle and a volume of $10 \mathrm{~mL} / \mathrm{kg}$ bw. Distilled water was used as a vehicle. The studied doses were estimated based on the results from our previous investigations with the respective plant extracts (Vasileva et al., 2016, 2017).

\subsection{Open field test}

The spontaneous exploratory behavior was evaluated in an openfield test (OFT) once after the first administration of the tested substances and after eight days of repeated treatment. Briefly, each rat was placed in the center of an automated activity cage apparatus (Ugo Basile, Italy) with infrared detectors and left for $5 \mathrm{~min}$ to explore freely the field. The horizontal (crossings) and vertical (rearings) movements were recorded. Prior to each trial to remove any olfactory cues the cage was cleaned thoroughly.

\subsection{Morris water maze}

The Morris water maze task (MWM) was used to evaluate the spatial orientation, learning, memory and cognitive flexibility. The water maze consists of a circular pool $(120 \mathrm{~cm}$ diameter and $60 \mathrm{~cm}$ depth), filled with water $\left(\mathrm{t}=22 \pm 1{ }^{\circ} \mathrm{C}\right)$ up to $45 \mathrm{~cm}$, which is divided into 4 quadrants with a transparent platform submerged $2 \mathrm{~cm}$ below the water surface. Each rat was trained for 5 consecutive days with 3 trials per day to escape the maze by locating the platform with the help of visual cues. Every learning trial started from a different pseudo-random position and lasted for maximal time of $120 \mathrm{~s}$ and additional $15 \mathrm{~s}$ on the platform for learning. The memory retention test was performed for maximal time of $60 \mathrm{~s} 3$ days after the last training session in order to assess the recall of memory traces. The escape latency, i.e. the time rodent takes to find the platform was used as an outcome measure (Vorhees and Williams, 2006).

\subsection{Lipopolysaccharide-induced inflammation}

On the 37th day of the study the immune reactivity and the development of depression-like behavior were tested in the LPS-induced inflammation model. Each animal was injected intraperitoneally with LPS in a dose of $250 \mu \mathrm{g} / \mathrm{kg}$ bw dissolved in a sterile normal saline (Elgarf et al., 2014; Vasileva et al., 2017).

\subsection{Serum and brain tissues sample preparation}

Four hours following the LPS injection blood samples were obtained from the rats' tail veins for estimation of the TNF- $\alpha$ and IL-6 levels in serum. The supernatant (i.e. serum sample) was achieved following clotting and centrifugation $\left(3000 \mathrm{rpm}, 4^{\circ} \mathrm{C}, 10 \mathrm{~min}\right)$ and subsequently frozen at $-80^{\circ} \mathrm{C}$ prior to use.

Immediately after obtaining the blood samples the animals were deeply anaesthetized with an intraperitoneal injection of pentobarbital sodium $(65 \mathrm{mg} / \mathrm{kg} \mathrm{bw})$. All animals were decapitated and the brain tissues were carefully removed, rapidly frozen and stored at $-80^{\circ} \mathrm{C}$ until estimation of the TNF- $\alpha$ concentration.

\subsection{ELISA detection of cytokines}

Commercially available ELISA kits were used to detect the concentrations of TNF- $\alpha$ in serum and brain tissue homogenate and IL- 6 in serum strictly following the manufacturer's recommendations. The optical density was detected at $450 \mathrm{~nm}$ with the reference filter at $620 \mathrm{~nm}$. Linear equations derived from the standard curves of the concentrations of each cytokine were used to calculate the individual sample concentrations (Vasileva et al., 2017).

\subsection{Statistical analysis}

SigmaPlot 11.0 statistical software was used for the data analysis. The Shapiro-Wilk test was applied as a normality test. For the between groups variability the one-way analysis of variance (ANOVA) for independent measures was employed followed by the Tukey HSD post hoc test. The comparison within groups was done using the ANOVA for repeated measures followed by the Bonferroni post hoc test. Data was presented as mean \pm standard error of the mean (SEM) and values of $\mathrm{p}<0.05$ were considered significant.

\section{Results}

\subsection{Salidroside and curcumin alleviated the CMS-induced anhedonic} behavior in GPT

Within the control group, the preference for a glucose solution dropped from $98 \%$ at baseline to $63 \%$ on the 15 th day and remained close to these values till the end of the study. The CMS model group showed a significantly $(\mathrm{p}<0.05)$ lower preference for the sweet solution relative to the control group on the 22nd, 29th and 36th day of the study which is representative for the established anhedonic-like state. The fluoxetine treated animals, used as a positive control with respect to the antidepressant activity, showed a statistically significant increased glucose preference $(\mathrm{p}<0.05)$ both after single and repeated administration while the groups treated respectively with salidroside $5 \mathrm{mg} / \mathrm{kg}$ bw, curcumin $20 \mathrm{mg} / \mathrm{kg}$ bw and their combination significantly increased the preference to glucose solution $(p<0.05)$ only after multiple administration on the 36th day compared to the CMS model group (Fig. 2).

\subsection{Body weight is affected by the CMS procedures, but not changed by the studied compounds}

The non-stressed controls showed a significant increase $(\mathrm{p}<0.05)$ in their weight from the 8th day on every scaling till the end of the study compared to baseline. The weight of the CMS model group did not change significantly throughout the experiment compared to its baseline measures. From the 15th day till the end of the study the weight of the CMS model group was significantly lower $(p<0.05)$

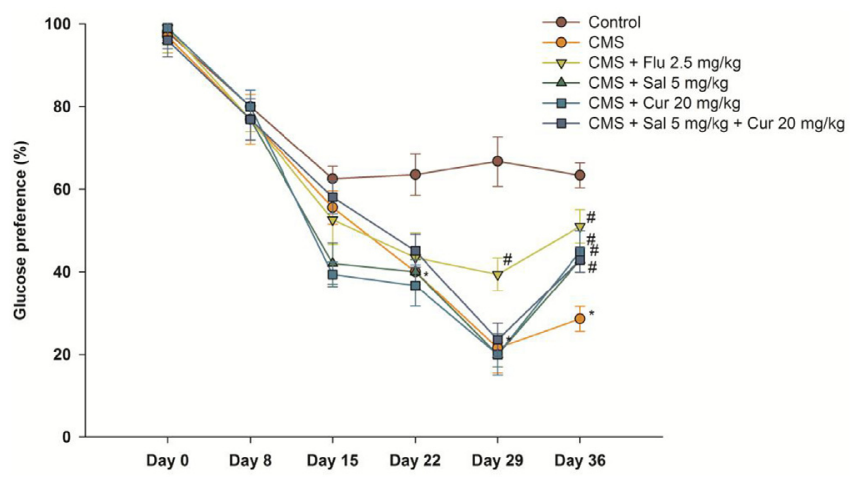

Fig. 2. Effect of salidroside, curcumin and their combination on the glucose preference in the GPT. All values were presented in means \pm SEM of eight animals per group and analyzed with the one-way ANOVA followed by the Tukey HSD post hoc test. ${ }^{*} \mathrm{p}<0.05$ as compared to the same day control group, ${ }^{\#} \mathrm{p}<0.05$ as compared to the same day CMS model group. 
Table 1

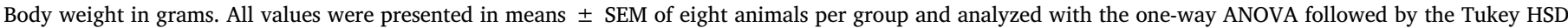

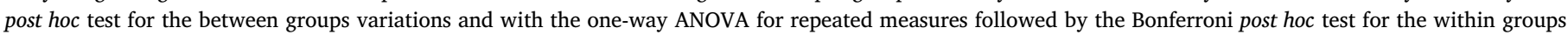
variations. ${ }^{*} \mathrm{p}<0.05$ as compared to day 0 of the control group, ${ }^{*} \mathrm{p}<0.05 \mathrm{CMS}$ model group compared to same day control group.

\begin{tabular}{|c|c|c|c|c|c|c|}
\hline & Day 0 & Day 8 & Day 15 & Day 22 & Day 29 & Day 36 \\
\hline Control group & $158.75 \pm 6.105$ & $175.625 \pm 6.299^{*}$ & $196.875 \pm 7.376^{*}$ & $215.625 \pm 6.439^{*}$ & $225.625 \pm 7.285^{*}$ & $240 \pm 7.50^{*}$ \\
\hline CMS model group & $149.38 \pm 5.382$ & $139.375 \pm 3.946^{\#}$ & $158.125 \pm 4.426^{\#}$ & $165.625 \pm 4.575^{\#}$ & $164.375 \pm 4.057^{\#}$ & $167.5 \pm 2.988^{\#}$ \\
\hline CMS + Fluoxetine $2.5 \mathrm{mg} / \mathrm{kg}$ & $148.75 \pm 4.407$ & $133.75 \pm 5.57$ & $151.25 \pm 3.75$ & $156.88 \pm 3.652$ & $158.13 \pm 4.719$ & $171.88 \pm 5.743$ \\
\hline CMS + Salidroside $5 \mathrm{mg} / \mathrm{kg}$ & $148.75 \pm 3.374$ & $147.5 \pm 3.273$ & $155 \pm 4.33$ & $161.25 \pm 5.57$ & $160.63 \pm 5.382$ & $168.75 \pm 5.65$ \\
\hline CMS + Curcumin $20 \mathrm{mg} / \mathrm{kg}$ & $143.75 \pm 3.239$ & $135 \pm 6.05$ & $146.88 \pm 5.17$ & $153.13 \pm 7.192$ & $156.88 \pm 8.708$ & $164.38 \pm 8.683$ \\
\hline CMS + Salidroside $5 \mathrm{mg} / \mathrm{kg}+$ Curcumin $20 \mathrm{mg} / \mathrm{kg}$ & $151.25 \pm 6.25$ & $141.25 \pm 6.731$ & $152.50 \pm 6.050$ & $160.63 \pm 6.777$ & $162.50 \pm 7.319$ & $174.38 \pm 8.986$ \\
\hline
\end{tabular}

compared to the respective day controls. The groups subjected to the CMS and treated with fluoxetine, salidroside, curcumin and the combination of the two natural compounds showed no significant differences in the body weight compared to the CMS model group (Table 1).

\subsection{Curcumin reversed the CMS-induced inhibition of the locomotor} activity in OFT after single and repeated administration suggesting a strong stimulatory effect

In the OFT on the 29th day, the CMS model group showed significantly reduced $(\mathrm{p}<0.05)$ horizontal and vertical movements compared to the controls. Animals subjected to the CMS and treated with curcumin showed significantly ( $p<0.05$ ) higher number of both crossings and rearings on the 29th and the 36th day as compared to the model group. The groups treated with fluoxetine, salidroside and combination of the two substances increased significantly $(\mathrm{p}<0.05)$ their horizontal and vertical movements after multiple administration on the OFT compared to the CMS group (Fig. 3A and B).

\subsection{Salidroside, curcumin and their combination improved the performance in MWM task in stressed rats and enhanced memory formation}

The control group significantly shortened the latency time for platform detection $(\mathrm{p}<0.05)$ across all training session days (i.e., from the 29th till the 33rd day) and at the memory test on the 36th day of the study compared to the first training trial. The CMS model group showed higher latency times compared to the respective day control group, but the differences were statistically significant $(p<0.05)$ at the second and the eighth day of the MWM test. The animals treated with fluoxetine significantly $(\mathrm{p}<0.05$ ) reduced the platform detection time in comparison with the model group during all days of the training session and on the memory test.

The salidroside treated animals significantly $(p<0.05)$ decreased the latency time compared to the CMS group on the second, fifth and eighth day of the MWM. The group treated with curcumin significantly

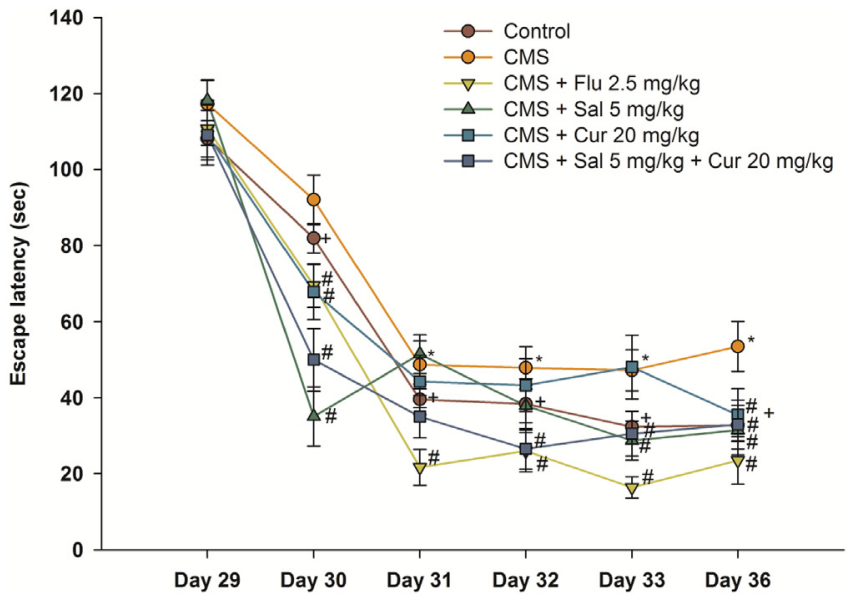

Fig. 4. Learning curve in the MWM task for assessment of the effect of salidroside, curcumin and their combination on the spatial learning and memory. All values were presented in means \pm SEM of eight animals per group and analyzed with the one-way ANOVA followed by the Tukey HSD post hoc test for the between groups variations and with the one-way ANOVA for repeated measures followed by the Bonferroni post hoc test for the within groups variations. ${ }^{+} \mathrm{p}<0.05$ as compared to the first training day of the control group, ${ }^{*} \mathrm{p}<0.05$ as compared to the same day of the control group; ${ }^{*} \mathrm{p}<0.05$ as compared to the same day of the CMS model group.

reduced the latency time $(\mathrm{p}<0.05)$ compared to the model group on the second day of the training session and on the memory test.

Animals treated with the combination of salidroside and curcumin showed a significant decrease in the platform detection time ( $\mathrm{p}<0.05$ ) compared to the model group on the second, fourth and fifth day of the training session and on the memory retention test (Fig. 4).
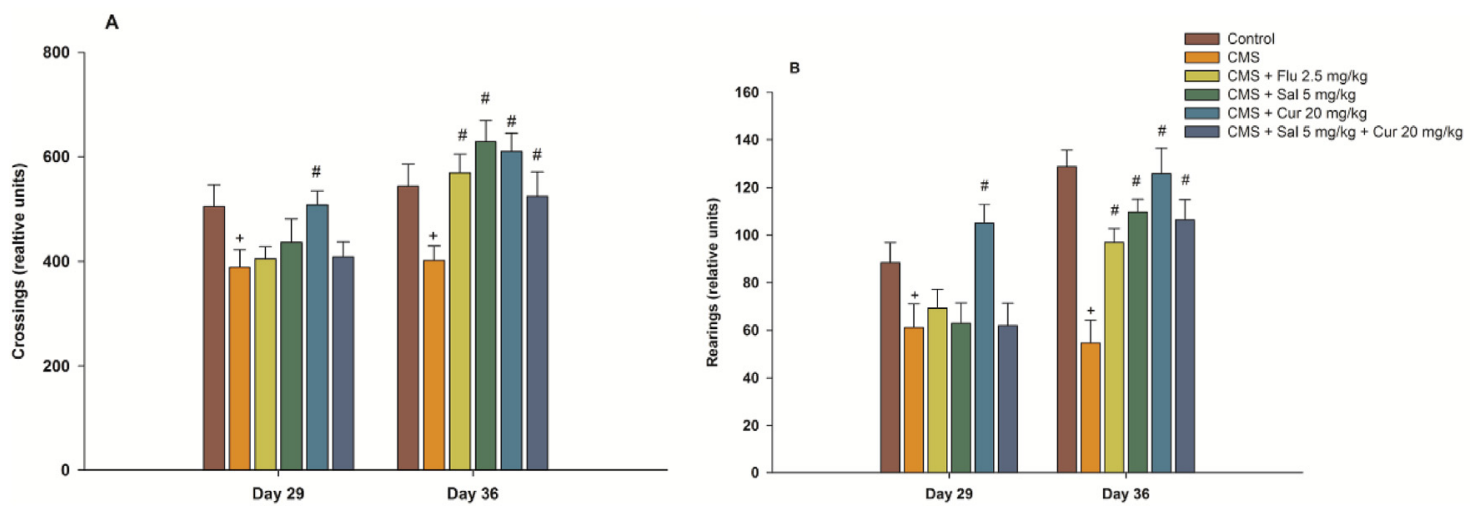

Fig. 3. Effects of salidroside, curcumin and their combination on the number of crossings (A) and rearings (B) registered in the OFT after single and multiple administrations. Data were expressed in means \pm SEM of eight animals per group and analyzed with the one-way ANOVA followed by the Tukey HSD post hoc test. ${ }^{+} \mathrm{p}<0.05$ as compared to the control group; ${ }^{\#} \mathrm{p}<0.05$ as compared to the CMS model group. 

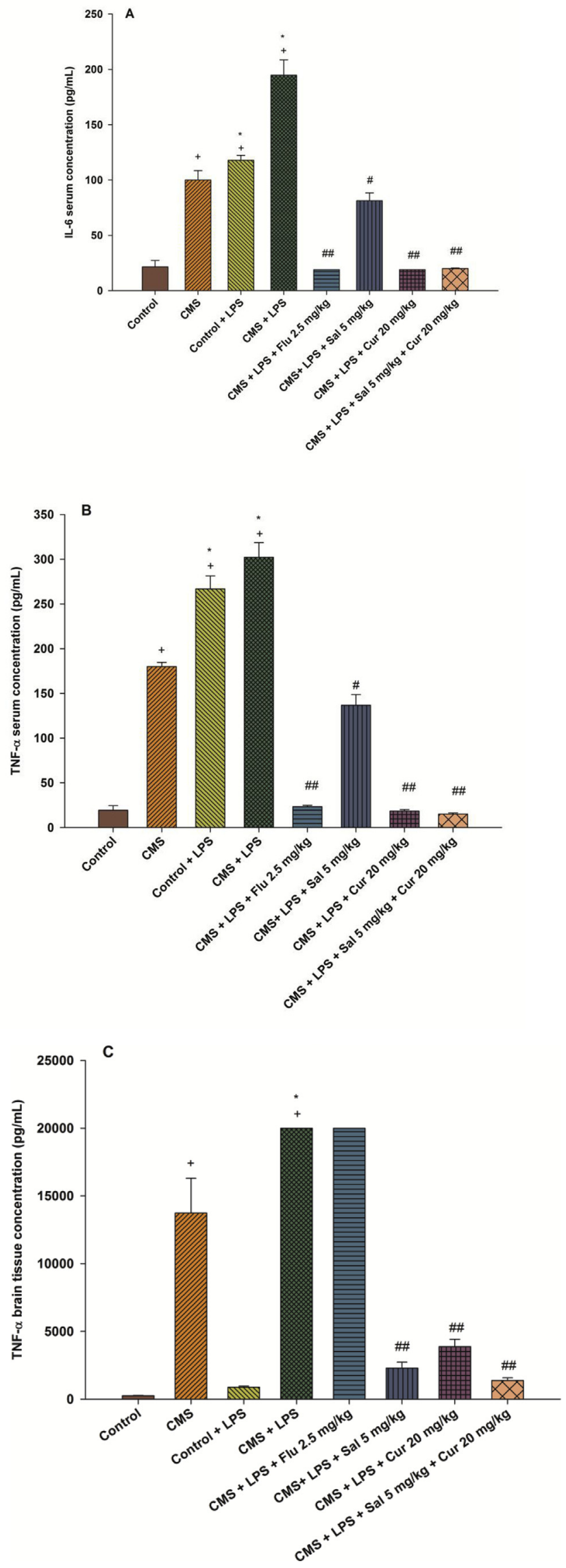

Fig. 5. Effects of salidroside, curcumin and their combination on the IL-6 levels in rats' sera (A), the TNF- $\alpha$ levels in rats' sera (B) and the TNF- $\alpha$ levels in rats' brain tissue homogenate (C) in $\mathrm{pg} / \mathrm{mL}$. All values were presented in means \pm SEM of eight animals per group and analyzed with the one-way ANOVA followed by the Tukey HSD post hoc test. ${ }^{+} \mathrm{p}<0.05$ as compared to the control group, ${ }^{*} \mathrm{p}<0.05$ CMS + LPS group as compared to the CMS model group, ${ }^{\#} \mathrm{p}<0.05$ as compared to the CMS + LPS group, ${ }^{\# \#} \mathrm{p}<0.01$ as compared to the CMS + LPS group.

3.5. Salidroside and curcumin prevented LPS-induced increase in cytokines' levels both in the serum and the brain tissue of stressed rats

\subsubsection{IL-6 levels in serum}

The combined model of the CMS followed by a LPS-challenge (CMS + LPS) significantly increased the levels of IL-6 (p < 0.05) in comparison to the CMS and the LPS alone. Furthermore, the IL- 6 levels in the CMS, the LPS and the CMS + LPS groups were all significantly higher $(\mathrm{p}<0.05)$ than in the controls.

The salidroside treated animals showed significantly $(\mathrm{p}<0.05)$ reduced IL- 6 compared to the CMS + LPS group. Furthermore, the groups treated with fluoxetine, curcumin and the combination of salidroside and curcumin showed significantly stronger $(\mathrm{p}<0.01)$ reduction in the IL-6 levels compared to the model CMS + LPS group (Fig. 5A).

\subsubsection{TNF- $\alpha$ levels in serum}

The serum levels of the TNF- $\alpha$ were significantly ( $\mathrm{p}<0.05$ ) elevated within the CMS + LPS model group in comparison to the CMS group. The CMS and the LPS model groups showed significantly $(\mathrm{p}<0.05)$ elevated serum levels of the TNF- $\alpha$ compared to the control group.

The animals treated with salidroside significantly $(\mathrm{p}<0.05)$ lowered the TNF- $\alpha$ levels compared to the animals from the CMS + LPS group. The groups treated respectively with fluoxetine, curcumin and the combination of the two natural compounds showed significantly decreased ( $\mathrm{p}<0.01$ ) TNF- $\alpha$ compared to the model CMS + LPS group (Fig. 5B).

\subsubsection{TNF- $\alpha$ levels in brain tissue}

Regarding the brain levels of TNF- $\alpha$, the rats from the combined CMS + LPS model group showed significant $(\mathrm{p}<0.05)$ increase in comparison with the CMS or the LPS groups, as well as, compared to the controls.

The rats treated with salidroside, curcumin and combination of both substances showed significantly decreased ( $\mathrm{p}<0.01$ ) TNF- $\alpha$ levels in brain tissue compared to the CMS + LPS group. The animals exposed to the combined CMS + LPS model treated with fluoxetine showed no significant changes in the TNF- $\alpha$ level compared to the model CMS + LPS group (Fig. 5C).

\section{Discussion}

The present study aimed to evaluate the effect of salidroside and curcumin in a CMS depression model followed by a LPS challenge on the development of depression-like behavioral state in rats and their immune response. Salidroside and curcumin are both derived from medicinal plants that are described with beneficial effects in depression and stress-induced disorders (Cropley et al., 2015; Vasileva et al., 2016; Xia et al., 2007).

The CMS model in all stressed animals resulted in an anhedonic-like behavior, disorders in the weight gain pattern and suppressed motor activity relative to the non-stressed controls. Within the first four weeks between the stressed groups there were no significant differences in the preference for glucose or the change in body weight due to the lack of treatment during this period. 
The state of anhedonia caused by the chronic stress model after single dose administration of the studied substances was influenced solely by fluoxetine. This rapid effect could be dedicated to the inhibition of the stress-induced corticosterone secretion reported for fluoxetine by other authors in the sucrose preference tests (Kubera et al., 2013). It is known that at least one week of application of fluoxetine is required to significantly affect the serotonin levels and to achieve the actual antidepressant action (Zou et al., 2013). Given to the short half-life of salidroside, it is likely that the application dose does not reach a sufficient concentration to show an anti-stress effect after single administration. A similar assumption could be made for the lack of effect of curcumin after single dose application. It can be speculated that with increase in the doses and changes in the mode of administration to ensure high bioavailability, these substances would exhibit a faster and stronger anti-stress effect after a single administration. Similar studies of salidroside, curcumin or their combination, evaluating the effect of single doses in stress or depression models were not found.

After repeated treatment for eight days, our results - with regard to the anhedonia - showed a significant antidepressant-like effect of the studied substances salidroside and curcumin comparable to that of fluoxetine. Repeated administration of fluoxetine for more than one week enhanced the antidepressant action by stimulating the serotonin mediation (Lu et al., 2017). Salidroside and curcumin presented an antidepressant-like effect, both administered either as monotherapy or in combination. In terms of the effect of salidroside in depression models, a recent study indicated antidepressant-like activity possibly due to modulation of the monoaminergic signaling pathways (Li et al., 2016). The action of curcumin to alleviate the anhedonic-like behavior in our study was possibly mediated by modulation of the HPA axis activity and the regulation of the stress hormones levels - mainly the corticosterone. The obtained results are in line with previous reports on strong antidepressant-like effect of repeated curcumin administration (10 mg/kg bw, via gavage) in a CMS model (Jiang et al., 2013; Liu et al., 2014). Furthermore, curcumin and its derivatives in stressed animals are known to decrease the serum corticosterone levels and modulate the sensitivity of serotonin receptors of type $5-\mathrm{HT}_{1 \mathrm{~A}}, 5-\mathrm{HT}_{1 \mathrm{~B}}$ and $5-\mathrm{HT}_{7}(\mathrm{Li}$ et al., 2009; Lian et al., 2018).

With regard to the locomotor activity our data confirmed that the CMS procedure suppresses both horizontal and vertical movements (Willner, 2005)., which was sustained after the stress protocol has been ceased. In our experiment, a single dose of curcumin increased both the crossings and the rearings which implied a rapid stimulatory effect on the overall motor activity, possibly through a sharp decrease in the stress hormones. Furthermore, the repeated administration resulted in eliminating the stress-induced impairment in the motor activity not only by curcumin, but also by fluoxetine and salidroside.

In experimental models of chronic stress and depression an atrophy of neurons and a reduced BDNF production were detected in the hippocampus and the prefrontal cortex in rodents (Liu et al., 2014; Rinwa et al., 2013). Depression has been characterized by a decrease in the BDNF levels in the hippocampus also in humans which leads to suppressed neurogenesis (Finnell and Wood, 2016). Except of the hippocampal impairment, the BNDF deficiency may also cause altered sensitivity of the glucocorticoid receptors (Sousa et al., 2008). Our previous data about the MWM task discussed its correlation with the impairment in the hippocampus (Vasileva et al., 2017) which was confirmed in the present study as the model of chronic stress provoked learning disabilities and memory impairments. The mitigation of such stress-induced alternations by salidroside and curcumin presented that these substances could also act at the hippocampal brain area and could modulate the BDNF levels in conditions of stress (Lian et al., 2018; Liu et al., 2014; Yang et al., 2014).

Salidroside and curcumin, as well as their combination, alleviated the stress-induced memory impairments after repeated administration, suggesting a memory-stimulating activity comparable to that of fluoxetine. In our study, fluoxetine improved the learning process and enhanced the recall of memory traces. The obtained data is in support to the found in the literature from other authors that showed the beneficial effect of fluoxetine on the spatial orientation and memory in the water maze task in non-stressed animals and in animals subjected to the chronic stress model (Gumuslu et al., 2013; Valluzzi and Chan, 2007).

Salidroside administered alone in our study stimulated the cognitive processes which is in line with the data of Yan et al. (2015) reporting similar effect in the water maze test on rats subjected to a model of brain hypoxia. The same authors have found that salidroside reduced the hypoxia-induced apoptosis in the hippocampus (Yan et al., 2015). Administration of salidroside in rats with an OBX depression model resulted in a significant increase in the BDNF levels, stimulated the resistance to hippocampal damage and regulated the glucocorticoid receptors' functions (Yang et al., 2014). These mechanisms, which protect against the hippocampal impairments, along with the modulation of the HPA axis activity and the anti-inflammatory properties of salidroside may explain its effectiveness in alleviating the cognitive symptoms of depression and chronic stress.

In terms of the effect of curcumin there are studies reporting that its chronic use improves learning and memory not only in an intact brain, but also under conditions of cognitive deficits (Ishrat et al., 2009; Reeta et al., 2010; Xu et al., 2007). Liu et al. (2014) assessed the effect on the memory and spatial orientation in a water maze test of curcumin administration in a chronic stress model. The authors suggested that the positive effect on the learning and memory of curcumin resulted from the increased levels of the BDNF and the extracellular signal-regulated kinase (ERK) in the hippocampus (Liu et al., 2014). In our study, curcumin appeared to improve the memory functions and when combined with salidroside, the beneficial effect on the spatial orientation and memory was found to be stronger.

The results of the ELISA assays for the two cytokines in the sera indicated similar tendencies as in our previous study (Vasileva et al., 2017), confirming that the combined CMS and LPS model induced robust immune response in the stressed animals. In the present experiment, to further investigate the role of the TNF- $\alpha$ in states of chronic stress or depression, its levels were also recorded in a brain tissue homogenate. Correlation between the TNF- $\alpha$ levels in the serum and the brain samples was observed, which revealed the significant difference between the control animals and the CMS model group. Inducing an immune response in the animals that have undergone the CMS procedure, by injecting the LPS, further increased the values of the studied cytokines confirming the hyperactivated immune function accompanying the depression-like state. After the eight days of repeated administration, the most pronounced anti-inflammatory effect was observed by fluoxetine and curcumin. For fluoxetine the inhibition of the production of pro-inflammatory cytokines is known from numerous preclinical and clinical studies (Kubera et al., 2011; Liu et al., 2011; Song et al., 2009). Maes (2001) suggested that at least partly the antidepressant effect of the SERTs was due to their immunomodulatory activity. In his study, depressed patients that administered therapy with conventional antidepressants such as the SERTs, the norepinephrine reuptake inhibitors (NETs) and the MAO-A inhibitors normalized their inflammatory immune response by decrease in the levels of IL- 6 and the acute phase proteins (Maes, 2001).

Curcumin in our study strongly suppressed the production of IL- 6 in the serum and the TNF- $\alpha$ in the serum and the brain tissue which is in line with data from other authors (Lopresti et al., 2014; Rinwa et al., 2013; Wang et al., 2014). Tizabi et al. (2014) reported a protective effect of curcumin in a LPS-induced inflammation model and suggested that the antidepressant properties were due to curcumin pronounced anti-inflammatory activity and the inhibition of the NO production. In depression models induced by multiple application of a LPS, curcumin exhibits antidepressant-like effect and suppress the cytokines production (Wang et al., 2014). Additionally, in a CMS model in rats the elevated levels of TNF- $\alpha$ and IL- 6 have been normalized by the chronic administration of curcumin at a dose of $10 \mathrm{mg} / \mathrm{kg}$ bw via gavage (Jiang 
et al., 2013).

Salidroside administered alone or in combination with curcumin also reduced the levels of the investigated cytokines both centrally and peripherally. These data suggested a possible immunomodulating activity of salidroside mediated by a moderate inhibition of the inflammatory response in chronic stress. The administration of salidroside in vitro has a positive effect on the immune response by stimulating both cellular and humoral immunity (Zhao et al., 2013). Lee et al. (2013) studied the production of the pro-inflammatory cytokines TNF$\alpha$, IL-6, IL-1 $\beta$ induced by LPS on microglial cells treated with salidroside, tyrosine, rosavin and the extract from $R$. rosea. The authors reported salidroside with the most potent anti-inflammatory activity in comparison to the other active components and the plant extract (Lee et al., 2013). In an OBX model of depression in rats, salidroside exhibits an anti-inflammatory effect as demonstrated by the reduction in the levels of the TNF- $\alpha$ and the IL-1 $\beta$ in the hippocampus (Yang et al., 2014).

The combined salidroside and curcumin treatment in our study showed strong anti-inflammatory effect on the LPS-induced immunoinflammatory response both peripherally and in the brain.

\section{Conclusions}

The groups exposed to the CMS procedure for four weeks demonstrated a variety of depression-like behavioral changes resembling an anhedonia, including a reduction in glucose preference, weight gain and locomotor activity compared to the non-stressed controls. As a summary of the behavioral tests' results following the model of chronic stress, the multiple administration of salidroside and curcumin both separately and in combination resulted in a pronounced antidepressantlike effect comparable to that of the conventional antidepressant fluoxetine. The strongest motor-stimulating effect was exhibited by curcumin.

In addition, the ELISA tests showed that CMS is affecting rats' immunity by increasing the levels of TNF- $\alpha$ and IL- 6 both peripherally and centrally. Treatment with salidroside, curcumin and their combination decreased the IL- 6 and the TNF- $\alpha$ levels suggesting potential synergic interaction of the two plant-derived compounds regarding their immunomodulatory, anti-inflammatory and anti-stress activities. These results indicated that the two molecules possess promising therapeutic potential for the treatment of stress-induced conditions and certain depressive-like or mood disorders.

\section{Conflicts of interest}

The authors declare that the present work is not a subject of any potential conflict of interest.

\section{Acknowledgments}

The project for establishment of CPSBB has received funding from the European Union's Horizon 2020 research and innovation programme under grant agreement № PlantaSYST - SGA/CSA: 739582 under FPA: 664620.

The authors declare that this work was supported by a grant from the Medical University of Plovdiv - Project SDP15/2015.

\section{Transparency document}

Transparency document related to this article can be found online at https://doi.org/10.1016/j.fct.2018.09.065.

\section{References}

Bortolato, B., Carvalho, A.F., Soczynska, J.K., Perini, G.I., McIntyre, R.S., 2015. The in volvement of TNF- $\alpha$ in cognitive dysfunction associated with major depressive disorder: an opportunity for domain specific treatments. Curr. Neuropharmacol. 13, 558-576.

Cropley, M., Banks, A.P., Boyle, J., 2015. The effects of Rhodiola rosea L. extract on anxiety, stress, cognition and other mood symptoms. Phyther. Res. 29, 1934-1939.

Dhabhar, F.S., 2014. Effects of stress on immune function: the good, the bad, and the beautiful. Immunol. Res. 58, 193-210.

Elgarf, A.-S. a., Aboul-Fotouh, S., Abd-Alkhalek, H. a., El Tabbal, M., Hassan, A.N., Kassim, S.K., Hammouda, G. a., Farrag, K. a., Abdel-tawab, A.M., 2014. Lipopolysaccharide repeated challenge followed by chronic mild stress protocol introduces a combined model of depression in rats: reversibility by imipramine and pentoxifylline. Pharmacol. Biochem. Behav. 126, 152-162.

Finnell, J.E., Wood, S.K., 2016. Neuroinflammation at the interface of depression and cardiovascular disease: evidence from rodent models of social stress. Neurobiol. Stress 4, 1-14.

Goel, A., Kunnumakkara, A.B., Aggarwal, B.B., 2008. Curcumin as "curecumin": from kitchen to clinic. Biochem. Pharmacol. 75, 787-809.

Gumuslu, E., Mutlu, O., Sunnetci, D., Ulak, G., Celikyurt, I.K., Cine, N., Akar, F., 2013. The effects of tianeptine, olanzapine and fluoxetine on the cognitive behaviors of unpredictable chronic mild stress-exposed mice. Drug Res. 63, 532-539.

Hatcher, H., Planalp, R., Cho, J., Torti, F.M., Torti, S.V., 2008. Curcumin: from ancient medicine to current clinical trials. Cell. Mol. Life Sci. 65, 1631-1652.

Hughes, M.M., Connor, T.J., Harkin, A., 2016. Stress-related immune markers in depression: implications for treatment. Int. J. Neuropsychopharmacol. 19, 1-19.

Hurley, L.L., Akinfiresoye, L., Nwulia, E., Kamiya, A., Kulkarni, A.A., Tizabi, Y., 2013. Antidepressant-like effects of curcumin in WKY rat model of depression is associated with an increase in hippocampal BDNF. Behav. Brain Res. 239, 27-30.

Ishrat, T., Hoda, M.N., Khan, M.B., Yousuf, S., Ahmad, M., Khan, M.M., Ahmad, A., Islam, F., 2009. Amelioration of cognitive deficits and neurodegeneration by curcumin in rat model of sporadic dementia of Alzheimer's type (SDAT). Eur. Neuropsychopharmacol $19,636-647$.

Jiang, H., Wang, Z., Wang, Y., Xie, K., Zhang, Q., Luan, Q., Chen, W., Liu, D., 2013 Antidepressant-like effects of curcumin in chronic mild stress of rats: involvement of its anti-inflammatory action. Prog. Neuro-Psychopharmacol. Biol. Psychiatry 47, 33-39.

Kubera, M., Curzytek, K., Duda, W., Leskiewicz, M., Basta-Kaim, A., Budziszewska, B., Roman, A., Zajicova, A., Holan, V., Szczesny, E., Lason, W., Maes, M., 2013. A new animal model of (chronic) depression induced by repeated and intermittent lipopolysaccharide administration for 4 months. Brain Behav. Immun. 31, 96-104.

Kubera, M., Grygier, B., Wrona, D., Rogóz, Z., Roman, A., Basta-Kaim, A., Budziszewska, B., Leskiewicz, M., Jantas, D., Nowak, W., Maes, M., Lason, W., 2011. Stimulatory effect of antidepressant drug pretreatment on progression of B16F10 melanoma in high-active male and female C57BL/6J mice. J. Neuroimmunol. 240-241, 34-44.

Lee, Y., Jung, J.-C., Jang, S., Kim, J., Ali, Z., Khan, I.A., Oh, S., 2013. Anti-inflammatory and neuroprotective effects of constituents isolated from Rhodiola rosea. Evid. Based. Complement. Alternat. Med. 2013, e514049.

Li, L., Qu, Y., Jin, X., Guo, X.Q., Wang, Y., Qi, L., Yang, J., Zhang, P., Li, L.Z., 2016. Protective effect of salidroside against bone loss via hypoxia-inducible factor-1a pathway-induced angiogenesis. Sci. Rep. 6, 32131.

Li, Y.C., Wang, F.M., Pan, Y., Qiang, L.Q., Cheng, G., Zhang, W.Y., Kong, L.D., 2009 Antidepressant-like effects of curcumin on serotonergic receptor-coupled AC-cAMP pathway in chronic unpredictable mild stress of rats. Prog. Neuro-Psychopharmacol. Biol. Psychiatry 33, 435-449.

Lian, L., Xu, Y., Zhang, J., Yu, Y., Zhu, N., Guan, X., Huang, H., Chen, R., Chen, J., Shi, G., Pan, J., 2018. Antidepressant-like effects of a novel curcumin derivative J147: involvement of 5-HT1A receptor. Neuropharmacology 135, 506-513.

Liu, D., Wang, Z., Gao, Z., Xie, K., Zhang, Q., Jiang, H., Pang, Q., 2014. Effects of curcumin on learning and memory deficits, BDNF, and ERK protein expression in rats exposed to chronic unpredictable stress. Behav. Brain Res. 271, 116-121.

Liu, D., Wang, Z., Liu, S., Wang, F., Zhao, S., Hao, A., 2011. Anti-inflammatory effects of fluoxetine in lipopolysaccharide (LPS)-stimulated microglial cells. Neuropharmacology 61, 592-599.

Lopresti, A.L., Maes, M., Maker, G.L., Hood, S.D., Drummond, P.D., 2014. Curcumin for the treatment of major depression: a randomised, double-blind, placebo controlled study. J. Affect. Disord. 167, 368-375.

Lu, Y., Ho, C.S., Liu, X., Chua, A.N., Wang, W., McIntyre, R.S., Ho, R.C., 2017. Chronic administration of fluoxetine and pro-inflammatory cytokine change in a rat model of depression. PloS One 12, e0186700.

Maes, M., 2001. The immunoregulatory effects of antidepressants. Hum. Psychopharmacol. 16, 95-103.

Maes, M., Mihaylova, I., Kubera, M., Ringel, K., 2012. Activation of cell-mediated immunity in depression: association with inflammation, melancholia, clinical staging and the fatigue and somatic symptom cluster of depression. Prog. NeuroPsychopharmacol. Biol. Psychiatry 36, 169-175.

Marchev, A.S., Dinkova-Kostova, A.T., György, Z., Mirmazloum, I., Aneva, I.Y., Georgiev, M.I., 2016. Rhodiola rosea L.: from golden root to green cell factories. Phytochemistry Rev. 15, 515-536.

McEwen, B.S., 2007. Physiology and neurobiology of stress and adaptation: central role of the brain. Physiol. Rev. 87, 873-904.

Ménard, C., Hodes, G.E., Russo, S.J., 2016. Pathogenesis of depression: insights from human and rodent studies. Neuroscience 321, 138-162.

Miller, A.H., Raison, C.L., 2015. The role of inflammation in depression: from evolutionary imperative to modern treatment target. Nat. Rev. Immunol. 16, 22-34.

Ohgi, Y., Futamura, T., Kikuchi, T., Hashimoto, K., 2013. Effects of antidepressants on alternations in serum cytokines and depressive-like behavior in mice after lipopolysaccharide administration. Pharmacol. Biochem. Behav. 103, 853-859.

Pariante, C.M., 2017. Why are depressed patients inflamed? A reflection on 20 years of 
research on depression, glucocorticoid resistance and inflammation. Eur Neuropsychopharmacol 27, 554-559.

Pleil, K.E., Lopez, A., McCall, N., Jijon, A.M., Bravo, J.P., Kash, T.L., 2012. Chronic stress alters neuropeptide y signaling in the bed nucleus of the stria terminalis in DBA/2J but not C57BL/6J mice. Neuropharmacology 62, 1777-1786.

Reeta, K.H., Mehla, J., Gupta, Y.K., 2010. Curcumin ameliorates cognitive dysfunction and oxidative damage in phenobarbitone and carbamazepine administered rats. Eur. J. Pharmacol. 644, 106-112.

Rinwa, P., Kumar, A., Garg, S., 2013. Suppression of neuroinflammatory and apoptotic signaling cascade by curcumin alone and in combination with piperine in rat model of olfactory bulbectomy induced depression. Plos One 8, e61052.

Shi, T.Y., Feng, S.F., Xing, J.H., Wu, Y.M., Li, X.Q., Zhang, N., Tian, Z., Liu, S.B., Zhao, M.G., 2012. Neuroprotective effects of salidroside and its analogue tyrosol galactoside against focal cerebral ischemia in vivo and $\mathrm{H} 2 \mathrm{O} 2$-induced neurotoxicity in vitro. Neurotox. Res. 21, 358-367.

Song, C., Halbreich, U., Han, C., Leonard, B.E., Luo, H., 2009. Imbalance between proand anti-inflammatory cytokines, and between Th1 and Th2 cytokines in depressed patients: the effect of electroacupuncture or fluoxetine treatment Pharmacopsychiatry 42, 182-188.

Sousa, N., Cerqueira, J.J., Almeida, O.F.X., 2008. Corticosteroid receptors and neuroplasticity. Brain Res. Rev. 57, 561-570.

Tizabi, Y., Hurley, L., Qualls, Z., Akinfiresoye, L., 2014. Relevance of the anti-inflammatory properties of curcumin in neurodegenerative diseases and depression. Molecules 19, 20864-20879.

Valluzzi, J.A., Chan, K., 2007. Effects of fluoxetine on hippocampal-dependent and hippocampal-independent learning tasks. Behav. Pharmacol. 18, 507-513.

Vasileva, L.V., Getova, D.P., Doncheva, N.D., Marchev, A.S., Georgiev, M.I., 2016. Beneficial effect of commercial Rhodiola extract in rats with scopolamine-induced memory impairment on active avoidance. J. Ethnopharmacol. 193, 586-591.

Vasileva, L.V., Saracheva, K.E., Ivanovska, M.V., Petrova, A.P., Murdjeva, M.A., GetovaSpasova, D.P., Sucouglu, E., Murdjeva, M.A., Getova-Spasova, D.P., 2017. Beneficial effect of chronic treatment with extracts from Rhodiola rosea L. and Curcuma longa L. on the immunoreactivity of animals subjected to a chronic mild stress model. Folia Med. (Plovdiv). 59, 95-104.

Vorhees, C.V., Williams, M.T., 2006. Morris water maze: procedures for assessing spatial and related forms of learning and memory. Nat. Protoc. 1, 848-858.
Wang, Z., Zhang, Q., Yuan, L., Wang, S., Liu, L., Yang, X., Li, G., Liu, D., 2014. The effects of curcumin on depressive-like behavior in mice after lipopolysaccharide administration. Behav. Brain Res. 274, 282-290.

Willner, P., 2017. The chronic mild stress (CMS) model of depression: history, evaluation and usage. Neurobiol. Stress 6, 78-93.

Willner, P., 2005. Chronic mild stress (CMS) revisited: consistency and behaviouralneurobiological concordance in the effects of CMS. Neuropsychobiology 52, 90-110.

Xia, X., Cheng, G., Pan, Y., Xia, Z.H., Kong, L.D., 2007. Behavioral, neurochemical and neuroendocrine effects of the ethanolic extract from Curcuma longa L. in the mouse forced swimming test. J. Ethnopharmacol. 110, 356-363.

Xu, Y., Ku, B., Cui, L., Li, X., Barish, P.A., Foster, T.C., Ogle, W.O., 2007. Curcumin reverses impaired hippocampal neurogenesis and increases serotonin receptor $1 \mathrm{~A}$ mRNA and brain-derived neurotrophic factor expression in chronically stressed rats. Brain Res. 1162, 9-18.

Yan, Z.-Q., Chen, J., Xing, G.-X., Huang, J.-G., Hou, X.-H., Zhang, Y., 2015. Salidroside prevents cognitive impairment induced by chronic cerebral hypoperfusion in rats. J. Int. Med. Res. 43, 402-411.

Yang, S.-J., Yu, H.-Y., Kang, D.-Y., Ma, Z.-Q., Qu, R., Fu, Q., Ma, S.-P., 2014. Antidepressant-like effects of salidroside on olfactory bulbectomy-induced pro-inflammatory cytokine production and hyperactivity of HPA axis in rats. Pharmacol. Biochem. Behav. 124, 451-457.

Zhang, J., Zhen, Y., Pu-Bu-Ci-Ren, Song, L., Kong, W., Shao, T., Li, X., Chai, X., 2013 Salidroside attenuates beta amyloid-induced cognitive deficits via modulating oxidative stress and inflammatory mediators in rat hippocampus. Behav. Brain Res. 244, $70-81$

Zhao, X., Lu, Y., Tao, Y., Huang, Y., Wang, D., Hu, Y., Liu, J., Wu, Y., Yu, Y., Liu, C., 2013 Salidroside liposome formulation enhances the activity of dendritic cells and immune responses. Int. Immunopharm. 17, 1134-1140.

Zhu, L., Chen, T., Chang, X., Zhou, R., Luo, F., Liu, J., Zhang, K., Wang, Y., Yang, Y., Long, H., Liu, Y., Yan, T., Ma, C., 2016. Salidroside ameliorates arthritis-induced brain cognition deficits by regulating Rho/ROCK/NF-кB pathway. Neuropharmacology 103, 134-142.

Zou, C., Ding, X., Flaherty, J., Dong, B., 2013. Clinical efficacy and safety of fuoxetine in generalized anxiety disorder in Chinese patients. Neuropsychiatric Dis. Treat. 9, 1661-1670. 